\title{
Prevalence of Overweight/Obesity and Its Associations with Hypertension, Diabetes, Dyslipidemia, and Metabolic Syndrome: A Survey in the Suburban Area of Beijing, 2007
}

\author{
Lei Zhang ${ }^{a, c, *} \quad$ Wei-Hong Zhang ${ }^{b, *} \quad$ Lian Zhang $^{a} \quad$ Pei-Yu Wang ${ }^{c}$ \\ ${ }^{a}$ Key Laboratory of Carcinogenesis and Translational Research (Ministry of Education), Department of Epidemiology, \\ Peking University Cancer Hospital \& Institute, Beijing, \\ ${ }^{b}$ Department of Basic Medicine, Nursing College, Zhengzhou University, Zhengzhou, \\ ${ }^{c}$ Department of Social Medicine and Health Education, School of Public Health, Peking University, Beijing, China
}

\section{Keywords}

Overweight · Obesity · Diabetes · Hypertension .

Dyslipidemia - Metabolic syndrome

\section{Summary}

Objective: This study aims to estimate the up-to-date prevalence of overweight/obesity in the suburban area of Beijing, China, and its associations with hypertension, diabetes, dyslipidemia, and metabolic syndrome (MetS). Methods: A cross-sectional survey in 19,003 suburban adults was carried out in Beijing, 2007. Overweight and obesity class 1,2 , and 3 were defined as a body mass index (BMI) of 25.0-29.9, 30.0-34.9, 35.039.9 , and $\geq 40 \mathrm{~kg} / \mathrm{m}^{2}$, respectively. Results: The agestandardized prevalence of overweight/obesity was $31.9 \%$ in the study. With increasing BMI, the prevalence of hypertension, diabetes, dyslipidemia, and MetS also increased. With normal body weight as reference, the adjusted odds ratio (95\% confidence interval) of hypertension for overweight and obesity class 1-3 was 2.5 $(2.2-2.7), 3.1(2.8-3.4), 3.6(3.1-4.3)$, and $4.5(4.0-5.2)$, respectively. The same trend was observed for diabetes and MetS. For dyslipidemia, however, participants with obesity class 2 had the highest odds ratio. Conclusion: The prevalence of hypertension, diabetes, dyslipidemia, and MetS increases with increasing BMI. Overweight/

*These authors contributed equally to this work. obesity is an important risk factor for these diseases. To reduce the social burden of these obesity-related diseases, effective strategies for the prevention of overweight/obesity should be implemented.

\section{Introduction}

With the rapid industrialization and urbanization in recent decades, the prevalence of overweight and obesity has increased significantly in China. Data from the China National Nutrition and Health Survey (CNNS) in 2002 indicates that the prevalence of overweight and obesity is 18.9 and $2.9 \%$, respectively, among adults aged 18 years and over, with overweight defined as a body weight index (BMI) of 25.0$29.9 \mathrm{~kg} / \mathrm{m}^{2}$ and obesity as a BMI of $30.0 \mathrm{~kg} / \mathrm{m}^{2}$ or greater [1]. Between 1992 and 2002, the prevalence of overweight and obesity increased by about 38.6 and $80.6 \%$, respectively, among Chinese residents, with a $40.7 \%$ increase in overweight detection rate and $97.2 \%$ increase in obesity prevalence among adults [1].

Overweight/obesity is an important modifiable risk factor for cardiovascular disease (CVD) and other chronic diseases [2-5]. Excess weight has been shown to be associated with increased prevalence of type 2 diabetes, hypertension, dyslipidemia, metabolic syndrome (MetS) and certain cancers; while weight loss dramatically reduces these obesity-related dis-

Pei-Yu Wang

Department of Social Medicine and Health Education School of Public Health, Peking University,

No.38, Xueyuan Road, Haidian District, 100191Beijing, China

Tel./Fax +861082802502

wpeiyu@hsc.pku.edu.cn 
eases $[6,7]$. These diseases have become the main cause of death worldwide [8]. In China, chronic diseases, most of which relate to overweight/obesity, account for $85 \%$ of all deaths in urban and $84 \%$ of deaths in rural areas [9]. An improved understanding of the associations between obesity and obesityrelated diseases may increase public awareness of the benefits of maintaining a healthy weight, which is of special importance for obesity prevention and treatment in areas where industrialization and urbanization are taking place. The aims of the present study were to i) provide the up-to-date prevalence estimates of overweight/obesity in individuals aged 18 years and over; ii) investigate the associations between overweight/obesity and the above obesity-related diseases in the suburban area of Beijing, China.

\section{Participants and Methods}

\section{Study Population}

A cross-sectional population-based survey by stratified multi-stage cluster sampling was conducted in the Haidian district suburban area of Beijing, 2007. In brief, a random 3-stage stratified sampling method was used to select a representative sample of the general population. In stage 1 , 5 towns were randomly selected. In the second stage of sampling, 30 villages/street districts were randomly selected from a total of 76 villages/ street districts. In the final stage, a total of 20,655 people aged 18 years and over were randomly chosen from these districts and invited to participate, whose age and sex distribution was similar to that of the total population. Finally, 19,003 people completed the anthropometric survey and laboratory examination and were included in the present study. The overall response rate was $92.0 \%$ (men $89.7 \%$, women $92.9 \%$ ).

\section{Data Collection}

Data collection was performed in local health centers or in community clinics in the participant's residential area. During the study visit, trained research staff members administered a standardized questionnaire to obtain the subject's information of age, sex, education, family history, physical activity, alcohol use, dietary habits, cigarette smoking, and the previous diagnosis and treatment of certain diseases including hypertension, high cholesterol, and diabetes. Body weight and height were measured twice during the interview. Weight was measured in light indoor clothing without shoes on electronic scales placed on a firm level surface to the nearest $0.1 \mathrm{~kg}$. Height was measured without shoes with a wall-mounted stadiometer to the nearest $0.1 \mathrm{~cm}$. Blood pressure was measured 3 times for participants in the seated position after at least 5 min of rest using a calibrated mercury sphygmomanometer. Participants were advised to avoid cigarette smoking, alcohol, caffeinated beverages, and exercise for at least 30 min before measurement.

\section{Laboratory Measurements}

Overnight fasting blood specimens were obtained by venipuncture for measurement of serum lipids and plasma glucose. Participants who did not meet overnight fasting were asked to visit centers later when their fasting time was more than $10 \mathrm{~h}$. Blood specimens were centrifuged and plasma was stored at $-80{ }^{\circ} \mathrm{C}$ until the laboratory assay. Plasma glucose was measured using a modified hexokinase enzymatic method (Hitachi Automatic Clinical Analyser, Model 7060, Tokyo, Japan). Concentrations of total cholesterol (TC), high density lipoprotein (HDL) cholesterol, and triglycerides (TG) were assessed enzymatically with commercially available reagents [10]. Lipid measurements were standardized according to the criteria of the Centers for Disease Control and Prevention - National Heart, Lung, and Blood Institute Lipid Standardization Program [11]. For participants with a TG level $<4.5 \mathrm{mmol} / \mathrm{l}$, low density lipoprotein (LDL) cholesterol levels were calculated from the Friedewald equation (LDL cholesterol $=\mathrm{TC}-\mathrm{HDL}$ cholesterol $-\mathrm{TG} / 5)$ [12]. All subjects had complete laboratory results.

\section{Criteria for Data Interpretation}

BMI was calculated as body weight in kilograms per square meters of height. Overweight and obesity class 1,2 , and 3 were defined as a BMI of $25.0-29.9,30.0-34.9,35.0-39.9$, and $\geq 40 \mathrm{~kg} / \mathrm{m}^{2}$, respectively [13]. In this study, we did not differentiate between normal weight and underweight class.

Hypertension was defined as an average (calculated from 3 measurements) systolic blood pressure (SBP) $\geq 140 \mathrm{~mm} \mathrm{Hg}$ and/or an average diastolic blood pressure (DBP) $\geq 90 \mathrm{~mm} \mathrm{Hg}$. Participants on self-reported regular antihypertensive treatment were considered to have hypertension [14].

Diabetes was diagnosed as having a fasting plasma glucose (FPG) level $\geq 7.0 \mathrm{mmol} / \mathrm{l}$ or the self-reported the previous diagnosis of diabetes, or current use of anti-diabetes medication [15].
Table 1. General characteristics of the study participants $^{\mathrm{a}}$

\begin{tabular}{lcccc}
\hline Characteristics & Total & Men & Women & p value $^{\mathrm{b}}$ \\
\hline Age, years & $48.0 \pm 6.9$ & $47.7 \pm 7.1$ & $48.2 \pm 6.9$ & $>0.05$ \\
Weight, $\mathrm{kg}$ & $68.0 \pm 11.2$ & $74.1 \pm 11.5$ & $64.5 \pm 11.4$ & $<0.01$ \\
$\mathrm{BMI}, \mathrm{kg} / \mathrm{m}^{2}$ & $23.7 \pm 3.3$ & $23.8 \pm 3.1$ & $23.6 \pm 3.0$ & $>0.05$ \\
$\mathrm{SBP}, \mathrm{mm} \mathrm{Hg}$ & $127.9 \pm 16.2$ & $129.5 \pm 15.9$ & $127.0 \pm 16.4$ & $<0.01$ \\
$\mathrm{DBP}, \mathrm{mm} \mathrm{Hg}$ & $83.7 \pm 11.9$ & $84.5 \pm 12.2$ & $80.5 \pm 11.5$ & $<0.01$ \\
FPG $\mathrm{mmol} / \mathrm{l}$ & $5.60 \pm 1.06$ & $5.56 \pm 1.05$ & $5.66 \pm 1.09$ & $<0.01$ \\
TC $\mathrm{mmol} / \mathrm{l}$ & $4.76 \pm 1.11$ & $4.74 \pm 1.14$ & $4.78 \pm 1.12$ & 0.02 \\
TG, $\mathrm{mmol} / \mathrm{l}$ & $1.61 \pm 0.55$ & $1.67 \pm 0.59$ & $1.58 \pm 0.54$ & $<0.01$ \\
HDL-C, $\mathrm{mmol} / \mathrm{l}$ & $1.37 \pm 0.38$ & $1.34 \pm 0.37$ & $1.39 \pm 0.35$ & $<0.01$ \\
LDL-C, mmol/l & $2.79 \pm 0.81$ & $2.76 \pm 0.77$ & $2.81 \pm 0.85$ & $<0.01$ \\
\hline
\end{tabular}

${ }^{a}$ Values are expressed as mean $\pm \mathrm{SD}$.

${ }^{b} \mathrm{p}$ values are from $\mathrm{t}$ test.

DBP = Diastolic blood pressure; FPG = fasting plasma glucose; HDL-C = high density lipoprotein cholesterol; LDL-C = low density lipoprotein cholesterol; SBP = systolic blood pressure; $\mathrm{TC}=$ total cholesterol; $\mathrm{TG}=$ triglycerides . 
Table 2. BMI distribution of the study participants by sex and age ${ }^{\mathrm{a}}$

\begin{tabular}{|c|c|c|c|c|c|}
\hline \multirow[t]{2}{*}{ Sex } & \multicolumn{5}{|l|}{ BMI, $\mathrm{kg} / \mathrm{m}^{2}$} \\
\hline & $\begin{array}{l}\text { normal } \\
(18.5-24.9)\end{array}$ & $\begin{array}{l}\text { overweight } \\
(25.0-29.9)\end{array}$ & $\begin{array}{l}\text { obesity class } 1 \\
(30.0-34.9)\end{array}$ & $\begin{array}{l}\text { obesity class } 2 \\
(35.0-39.9)\end{array}$ & $\begin{array}{l}\text { obesity class } 3 \\
(\geq 40.0)\end{array}$ \\
\hline Total $^{\mathrm{b}}$ & $62.7(11,915)$ & $24.2(4,597)$ & $4.6(872)$ & $2.1(407)$ & $1.0(190)$ \\
\hline Men overall $^{\mathrm{b}}$ & $63.2(4,518)$ & $25.5(1,823)$ & $4.4(315)$ & $1.7(122)$ & $0.8(60)$ \\
\hline 18-29 years & $66.0(388)$ & $21.5(127)$ & $3.9(20)$ & $1.4(7)$ & $0.5(2)$ \\
\hline 30-39 years & $65.3(750)$ & $24.0(289)$ & $4.2(44)$ & $1.6(17)$ & $0.8(8)$ \\
\hline 40-49 years & $61.9(1,560)$ & 25.9 (667) & $5.0(121)$ & $2.0(49)$ & $1.2(27)$ \\
\hline 50-59 years & $61.9(1,112)$ & $26.0(483)$ & $4.9(84)$ & $1.9(33)$ & $1.0(16)$ \\
\hline $60-69$ years & $65.1(466)$ & $22.3(176)$ & $4.7(32)$ & $1.7(11)$ & $0.7(5)$ \\
\hline$\geq 70$ years & $64.2(242)$ & $20.1(81)$ & $4.1(14)$ & $1.6(5)$ & $0.7(2)$ \\
\hline Women overall ${ }^{\mathrm{b}}$ & $62.4(7,397)$ & $23.4(2,774)^{\mathrm{c}}$ & $4.7(557)^{\mathrm{c}}$ & $2.4(285)^{\mathrm{c}}$ & $1.1(130)$ \\
\hline 18-29 years & $66.9(472)$ & $18.8(133)$ & $4.1(27)$ & $1.9(13)$ & $0.6(4)$ \\
\hline 30-39 years & $63.8(1,397)$ & $21.5(471)$ & $4.7(97)$ & $2.3(50)$ & $1.0(21)$ \\
\hline 40-49 years & $60.1(2,351)$ & $25.3(991)$ & $5.3(199)$ & 2.7 (105) & $1.3(51)$ \\
\hline 50-59 years & $61.5(1,956)$ & $24.2(770)$ & $5.1(155)$ & $2.6(83)$ & $1.2(38)$ \\
\hline $60-69$ years & $65.3(871)$ & $22.3(298)$ & $4.6(58)$ & $1.9(25)$ & $0.9(12)$ \\
\hline$\geq 70$ years & $65.6(350)$ & 20.7 (111) & $4.2(21)$ & $1.6(9)$ & $0.7(4)$ \\
\hline
\end{tabular}

${ }^{\mathrm{a}}$ Values are expressed as percentages (n).

${ }^{\mathrm{b}}$ Age-standardized prevalence rate. ${ }^{\mathrm{c}} \mathrm{p}<0.05$.

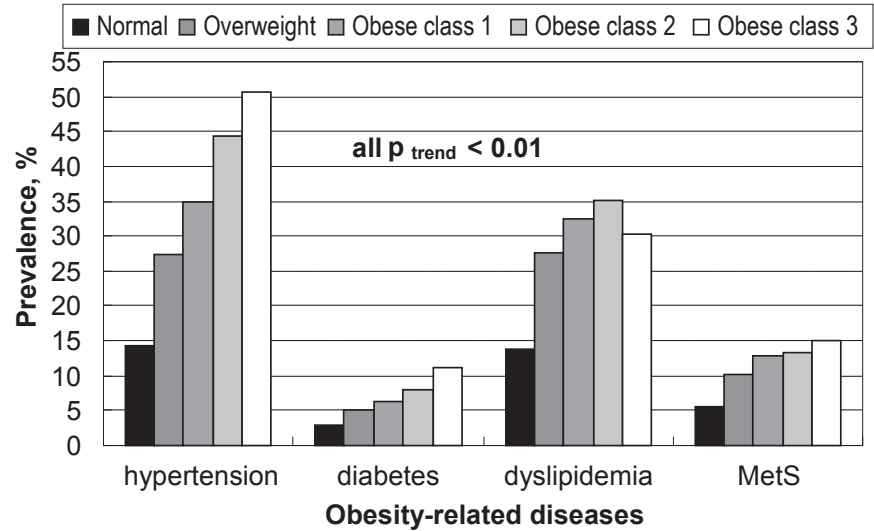

Fig. 1. The age and sex standardized prevalence of obesity-related diseases by BMI classification.

Dyslipidemia included any one of the following: $\mathrm{TC} \geq 5.2 \mathrm{mmol} / \mathrm{l}, \mathrm{TG} \geq$ $1.7 \mathrm{mmol} / \mathrm{l}, \mathrm{HDL}$ cholesterol $<1.0 \mathrm{mmol} / \mathrm{l}, \mathrm{LDL}$ cholesterol $\geq 3.4 \mathrm{mmol} / \mathrm{l}$. Dyslipidemia also included participants who reported treatment with cholesterol-lowering medication [11].

According to the criteria from the China Diabetes Society (CDS), MetS was defined as the presence of 3 or more of the following risk factors: $\mathrm{BMI} \geq 25 \mathrm{~kg} / \mathrm{m}^{2}$; serum TG $\geq 1.7 \mathrm{mmol} / 1$ and/or HDL cholesterol $<0.9 \mathrm{mmol} / \mathrm{l}$ in men or $<1.0 \mathrm{mmol} / 1$ in women; blood pressure $\geq$ $140 / 90 \mathrm{~mm} \mathrm{Hg}$ or patients with hypertension; or serum glucose concentration $\geq 6.1 \mathrm{mmol} / \mathrm{l}$ or patients with diabetes [16].

\section{Statistical Analysis}

Data were analyzed using SAS (version 9.1, 2005, SAS Institute Inc, Cary, NC). Descriptive statistics, such as means for continuous variables and their $95 \%$ confidence interval $(\mathrm{CI})$, frequencies for categorical variables were computed. Differences in continuous variables were tested using $t$ tests for independent samples. Prevalence of overweight/obesity by sex was compared using $\chi^{2}$ test, and prevalence trends across age group were analyzed using trend $\chi^{2}$ test. PROC SURVEYFREQ was used to obtain the prevalence of hypertension, diabetes, dyslipidemia, and MetS by different BMI, age and sex standardization was performed with the China 2000 Census population as the standard population. Odds ratio (OR) with $95 \%$ CI adjusted for age, sex, smoking, drinking, and other potential confounders was calculated by multivariate logistic regression to estimate the associations of hypertension, diabetes, dyslipidemia and MetS with overweight/obesity. Statistical significance was established at $\mathrm{p}<0.05$.

\section{Results}

General characteristics of the study participants are shown in table 1. A total of 19,003 people (7,148 men and 11,855 women) aged 18 years and over were included in this study. The average age of all participants was 48.0 (95\% CI 46.549.6) years old. The average BMI was 23.7 (95\% CI 23.2-24.3) $\mathrm{kg} / \mathrm{m}^{2}$. The average age and BMI between men and women had no statistically significant difference $(p>0.05)$. Men had higher body weight, SBP, DBP, and TG (all $\mathrm{p}<0.05$ ). Conversely, women had higher FPG, TC, LDL cholesterol, and HDL cholesterol (all $\mathrm{p}<0.05$ ).

As shown in table 2, the age-standardized prevalence of overweight/obesity was $31.9 \%$ (24.2\% for overweight and $7.7 \%$ for obesity). Although there was no significance in the overall prevalence of overweight/obesity between different genders $(32.4 \%$ in men and $31.6 \%$ in women), the prevalence of overweight was significantly higher and the prevalence of 
Fig. 2. Multivariate logistic regression adjusted odds ratio (adjusted for age, sex, drinking, smoking, physical activity) of obesity-related diseases by $\mathrm{BMI}(\mathrm{OW}=$ overweight, $\mathrm{Ob}=$ obesity class).

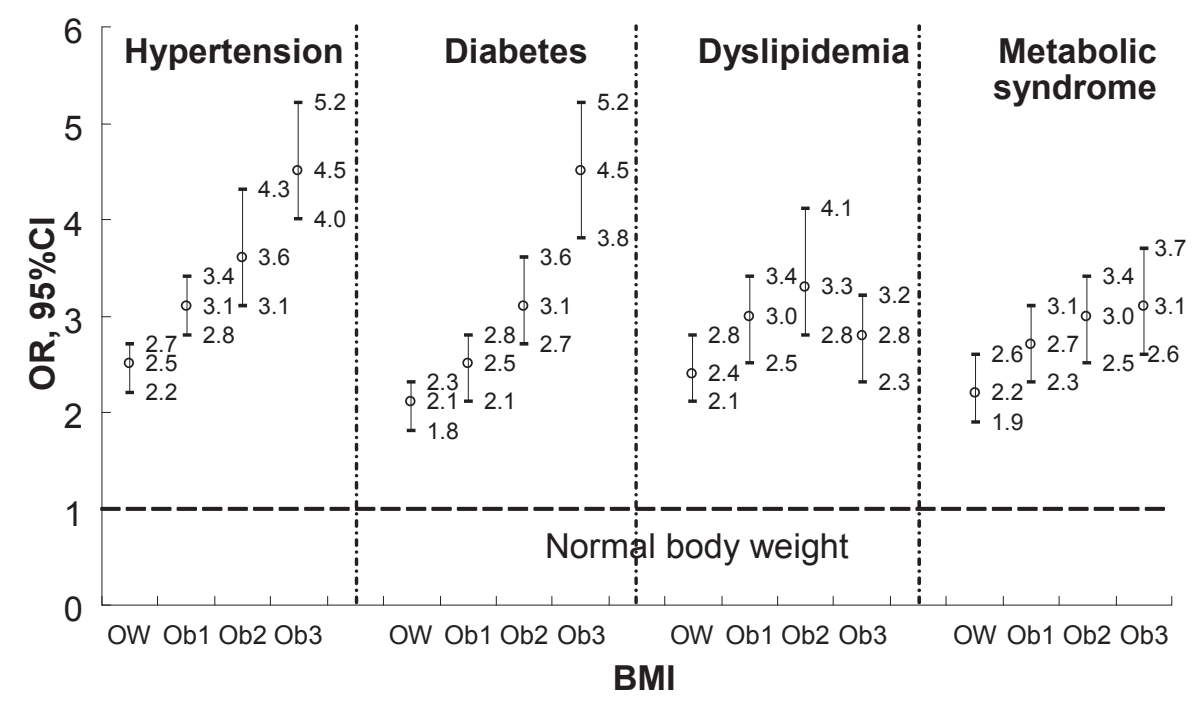

obesity class 2 and 3 was significantly lower in men than in women (all p < 0.05). Prevalence of overweight and obesity class $1-3$ increased in groups age $<50$ years old, and decreased in groups age $\geq 40$ years old among men and women $($ all $\mathrm{p}<0.05)$.

In general, the prevalence of hypertension, diabetes, dyslipidemia, and MetS was 33.6, 6.1, 30.3, and $11.6 \%$, respectively. The prevalence of these conditions by BMI is shown in figure 1 . The test for trend showed a significant increase of prevalence with increasing BMI classification of these obesity-related diseases. The most common obesity-related disease was hypertension, followed by dyslipidemia, MetS, and diabetes. For hypertension, the prevalence was 27.5, 34.8, 44.3 , and $50.7 \%$ in the participants with overweight and obesity class $1-3$, respectively. The same trend was observed for diabetes and MetS. It is notable that the highest prevalence of dyslipidemia was in the participants with obesity class 2 (35.1\%), instead of obesity class $3(30.4 \%)$.

The adjusted OR $(95 \% \mathrm{CI})$ was calculated by multivariate logistic regression to estimate the associations between obesity-related diseases and BMI (fig. 2). For hypertension, the participants with overweight and obesity class 1-3 had an adjusted OR of 2.5 (2.2-2.7), 3.1 (2.8-3.4), 3.6 (3.1-4.3), and 4.5 (4.0-5.2), respectively, with normal body weight as reference. The same trend was observed for diabetes and MetS, where the participants with obesity class 3 had the highest OR of 4.5, (3.8-5.2) and 3.1 (2.6-3.7), respectively. For dyslipidemia, however, the participants with obesity class 2 had the highest odds ratio of 3.3 (2.8-4.1).

\section{Discussion}

In this population-based cross-sectional study, the age-standardized prevalence of overweight/obesity was $31.9 \%$ in the suburban areas of Beijing. The prevalence in our study was notably high compared with that from CNNS data under the same criteria [1]. A possible explanation is the different area from which participants were recruited. Beijing as the capital is one of the biggest metropolises in China, and the suburban area of Beijing is experiencing rapid industrialization and urbanization which has dramatically changed the individual lifestyle. The previous CNNS study also demonstrated the high prevalence of overweight/obesity in Northern China including Beijing [17, 18]. Thus, the relatively high prevalence in our study is not surprising. On the other hand, our study was conducted in 2007. The high prevalence reflects, at least in part, the real increase in prevalence from 2002 when the CNNS study was conducted to 2007 when our study was conducted. Hence, a population-based nationwide survey for obesity should be carried out to update this data.

Several surveys with a relatively small sample size have been conducted in China. For example, one study from the metropolis of Shanghai found that the prevalence of overweight/obesity $\left(\mathrm{BMI} \geq 25 \mathrm{~kg} / \mathrm{m}^{2}\right)$ was $44.6 \%$ among adults aged 40 years and over [5]. Another study from Beijing and Shanghai provided a prevalence as high as $53.4 \%$ in adults aged 50-70 years when overweight/obesity was classified as $\mathrm{BMI} \geq 24 \mathrm{~kg} / \mathrm{m}^{2}$ [18]. The high prevalence in both studies was due to the higher age and/or lower criteria for overweight/ obesity. Some studies suggested that a BMI cut-off of $24 \mathrm{~kg} / \mathrm{m}^{2}$ might be appropriate for the criteria of overweight in China $[3,18]$. In our study, the cut-off of $25 \mathrm{~kg} / \mathrm{m}^{2}$ was used because the WHO Expert Consultation [19] agreed that this cut-off point should be maintained for international comparison. Data also show the prevalence of overweight/obesity in US adults aged 18 years and over to be $66.3 \%$ [20]. In comparison, the prevalence observed in China was notably low. However, this is not a reason to ignore this issue because prevalence in China is increasing and has an impact on many 
chronic diseases. Overweight and obesity have been consistently associated with the risk of CVD as well as other chronic conditions such as hypertension, diabetes, dyslipidemia, and MetS [2-7]. The health consequences of overweight and obesity have become the main problem of public health worldwide.

In the present study, the participants showed the highest prevalence of hypertension among the observed obesityrelated diseases. Its prevalence increased with increasing BMI and the prevalence was 4.7 -fold higher in the participants with obesity class $3\left(\mathrm{BMI} \geq 40 \mathrm{~kg} / \mathrm{m}^{2}\right)$ than in the participants with non-overweight/obesity. Our study was consistent with previous studies from China and other countries [4, 5, 21, 22]. For example, participants with obesity (BMI $\geq 25 \mathrm{~kg} / \mathrm{m}^{2}$ ) had an adjusted OR of 2.5 (1.9-3.1) with normal body weight as reference in a study with adults aged 40 years and over in Shanghai, China [5]. A US study collected data from participants aged 18 years and over and used the same criteria for overweight/obesity as our study [4]. Interestingly, the participants with overweight and obesity class 1 had a higher OR in our study than in the US study (2.5 vs. 1.7 for overweight; 3.1 vs. 2.6 for obesity class 1 ), suggesting that the impact of BMI on hypertension may be greater in the Chinese population. Since hypertension is an important risk factor for CVD and diabetes $[23,24]$, lower BMI cut-off might be appropriate to use as a threshold for public health action in the Chinese population.

The prevalence of diabetes was $6.1 \%$ in our study, which is the same as shown in participants aged 20-74 years from Qingdao City, China [25]. The prevalence increased with increasing BMI, which was consistent with previous studies [4, $5,22]$. In other words, obesity is a risk factor for diabetes, and this risk factor becomes stronger for extreme obesity. Similar to hypertension, participants with overweight and obesity class 1 in our study had a higher OR for diabetes than observed in the US study (2.1 vs. 1.2 for overweight; 2.5 vs. 1.6 for obesity class 1) [4]. It has been reported that there is a high prevalence of diabetic patients who remain undiagnosed in Mainland China [26], and obese participants are no more likely to have their diabetes diagnosed than normal-weight participants [27]. Thus, the diagnosis and treatment of diabetic patients from obese populations is urgently needed to decrease the social burden of this obesity-related disease.

In the present study, the highest prevalence of dyslipidemia was found in participants with obesity class 2 , instead of obesity class 3 . Other studies also showed that the increase in the prevalence of dyslipidemia peaked for individuals with obesity class 2 and hit a plateau for obesity class 3 individuals; they found that the prevalence of dyslipidemia increases until BMI surpasses $30 \mathrm{~kg} / \mathrm{m}^{2}$ and decreases with BMI $>40 \mathrm{~kg} / \mathrm{m}^{2}$ $[4,22]$. This was considered to be linked to the conservative definition of dyslipidemia including the following criteria: TC $\geq 6.22 \mathrm{mmol} / \mathrm{l}, \mathrm{TG} \geq 2.26 \mathrm{mmol} / \mathrm{l}$, HDL cholesterol $<1.04$ $\mathrm{mmol} / \mathrm{l}, \mathrm{LDL}$ cholesterol $\geq 4.14 \mathrm{mmol} / \mathrm{l}$ [21]. However, the definition in our study $(\mathrm{TC} \geq 5.2 \mathrm{mmol} / \mathrm{l}, \mathrm{TG} \geq 1.7 \mathrm{mmol} / \mathrm{l}$, HDL cholesterol $<1.0 \mathrm{mmol} / \mathrm{l}, \mathrm{LDL}$ cholesterol $\geq 3.4 \mathrm{mmol} / \mathrm{l}$ ) was less conservative. Thus, a further study should be carried to explore this unanticipated phenomenon.

MetS has become a major public health issue worldwide. The prevalence of MetS in a cross-sectional survey of 15,540 Chinese adults aged $35-74$ years was $9.8 \%$ in men and $17.8 \%$ in women [16]. In another study conducted in China with 10,326 participants aged 50-85 years, the prevalence was $15.6 \%$ [28]. Caution should be used in direct comparisons because those 2 studies selected older participants and used the definition of the US National Cholesterol Education Program (NCEP) instead of CDS. Similar to hypertension and diabetes, an increasing overweight and obesity class was associated with increased prevalence of MetS in our study and the study from the USA [4].

A limitation of our study is its reliance on estimates derived from a cross-sectional study. Cross-sectional study design does not allow for quantification of the importance of overweight/obesity in the incidence of these obesity-related conditions, which limits our ability to comment on causal relationships between overweight/obesity and the incidence of these conditions. However, previous studies have demonstrated the importance of overweight/obesity for the development of obesity-related conditions. Nevertheless, a further prospective study can be considered among the population in the future. A second limitation is that the applicability of diagnostic thresholds for overweight/obesity may be questioned. Despite some previous reports that $\mathrm{BMI} \geq 24 \mathrm{~kg} / \mathrm{m}^{2}$ may best define overweight/obesity among Chinese adults, we chose BMI $\geq 25 \mathrm{~kg} / \mathrm{m}^{2}$ to defined overweight/obesity for the current analysis to provide a prevalence of overweigh/obesity that is comparable to other studies and populations (such as Europeans, Japanese, and Americans).

\section{Conclusion}

In summary, findings from the present study showed an association between increasing BMI and an increase in the prevalence of hypertension, diabetes, dyslipidemia, and MetS. Overweight/obesity is an important risk factor for these obesity-related diseases. Some programs for body weight control should be initiated to combat these obesity-related conditions and reduce the overwhelming burden on the Chinese health care system.

\section{Disclosure Statement}

The authors declare no conflict of interests. 


\section{References}

1 National Center for Cardiovascular Diseases, China: Report on Cardiovascular Diseases in China. 1st ed. Beijing, Encyclopedia of China Publication, 2008.

2 Poirier P, Giles TD, Bray GA, Hong Y, Stern JS, Pi-Sunyer FX, et al: Obesity and cardiovascular disease: pathophysiology, evaluation, and effect of weight loss: an update of the 1997 American Heart Association Scientific Statement on Obesity and Heart Disease from the Obesity Committee of the Council on Nutrition, Physical Activity, and Metabolism. Circulation 2006;113:898-918.

3 Li R, Lu W, Jia J, Zhang S Shi L, Li Y, et al: Relationships between indices of obesity and its cardiovascular comorbidities in a Chinese population. Circ J 2008;72:973-978.

4 Nguyen NT, Magno CP, Lane KT, Hinojosa MW, Lane JS: Association of hypertension, diabetes, dyslipidemia, and metabolic syndrome with obesity: findings from the National Health and Nutrition Examination Survey, 1999 to 2004. J Am Coll Surg 2008:207:928-934.

5 Hu D, Xie J, Fu P, Zhou J, Yu D, Whelton PK, et al: Central rather than overall obesity is related to diabetes in the Chinese population: The InterASIA Study. Obesity 2007;15:2809-2816.

6 Hill AM, Kris-Etherton PM: Contemporary strategies for weight loss and cardiovascular disease risk factor modification. Curr Atheroscler Rep 2008;10: 486-496.

7 Sjöström L, Lindroos AK, Peltonen M, Torgerson J, Bouchard C, Carlsson B, et al: Lifestyle, diabetes, and cardiovascular risk factors 10 years after bariatric surgery. N Engl J Med 2004;351:2683-2693.

$>8$ Ogden CL, Yanovski SZ, Carroll MD, Flegal KM: The epidemiology of obesity. Gasroenterology 2007; 132:2087-2102.

9 Center for Statistics and Information, $\mathrm{MOH}$ : The Report of National Health Service Survey. 1st ed. Beijing, Publishing Company of Xiehe Medical University, 2004.

10 Allain CC, Poon LS, Chan CS, Richmond W, Fu PC: Enzymatic determination of total serum cholesterol. Clin Chem 1974;20:470-475.
1 Myers GL, Cooper GR, Winn CL, et al: The Centers for Disease Control - National Heart, Lung and Blood Institute Lipid Standardization Program. An approach to accurate and precise lipid measurements. Clin Lab Med 1989;9:105-135.

12 Friedewald WT, Levy RI, Fredrickson DS: Estimation of the concentration of low-density lipoprotein cholesterol in plasma, without use of the preparative ultracentrifuge. Clin Chem 1972;18:499-502.

13 Chobanian AV, Bakris GL, Black HR, Cushman WC, Green LA, Izzo JL Jr, et al: Seventh Report of the Joint National Committee on the Prevention, Detection, Evaluation, and Treatment of High Blood Pressure (JNC 7): resetting the hypertension sails. Hypertension 2003;41:1178-1179.

14 The Expert Committee on the Diagnosis and Classification of Diabetes Mellitus: Report of the expert committee on the diagnosis and classification of diabetes mellitus. Diabetes Care 2003;26:S5-S20.

15 Myers GL, Cooper GR, Winn CL, Smith SJ: The Centers for Disease Control - National Heart, Lung and Blood Institute Lipid Standardization Program. An approach to accurate and precise lipid measurements. Clin Lab Med 1989;9:105-135.

16 The Cooperation Group of the Chinese Medical Association Diabetes Branch Studying on the Metabolic Syndrome: Suggestion for the metabolic syndrome by the Chinese Medical Association Diabetes Branch. Chin J Diabetes 2004;12:156-161.

17 Gu D, Reynolds K, Wu X, Chen J, Duan X, Reynolds RF, et al: Prevalence of the metabolic syndrome and overweight among adult in China. Lancet 2005;365:1398-1405.

$18 \mathrm{Yu}$ Z, Lin X, Haas JD, Franco OH, Rennie KL, $\mathrm{Li} \mathrm{H}$, et al: Obesity related metabolic abnormalities: distribution and geographic differences among middle-aged and older Chinese populations. Prev Med 2009;48:272-278

19 WHO Expert Consultation: Appropriate bodymass index for Asian populations and its implications for policy and intervention strategies. Lancet 2004:363:157-163.
0 Nguyen NT, Magno CP, Lane KT, Hinojosa MW, Lane JS: Association of hypertension, diabetes, dyslipidemia, and metabolic syndrome with obesity: findings from the National Health and Nutrition Examination Survey, 1999 to 2004. J Am Coll Surg 2008;207:928-934.

21 Sun Z, Zheng L, Xu C, Li J, Zhang X, Liu S, et al Prevalence of pre-hypertension, hypertension and associated risk factors in Mongolian and Han Chinese populations in Northeast China. Int J Cardiol 2008;128:250-254.

22 Bays HE, Chapman RH, Grandy S: The relationship of body mass index to diabetes mellitus, hypertension, and dyslipidemia: comparison of data from two national surveys. Int J Clin Pract 2007;61: 737-747.

23 Glasser SP: Hypertension syndrome and cardiovascular events. High blood pressure is only one risk factor. Postgrad Med 2001;110:29-36.

24 Sun Z, Zheng L, Xu C, Zhang X, Li J, Liu S, et al: Prevalence of diabetes and impaired fasting glucose in hypertensive adults in rural China. Acta Cardiol 2009;64:351-356.

25 Dong Y, Gao W, Nan H, Yu H, Li F, Duan W, et al: Prevalence of type 2 diabetes in urban and rural Chinese populations in Qingdao, China. Diabet Med 2005;22:1427-1433.

26 Wong KC, Wang Z: Prevalence of type 2 diabetes mellitus of Chinese populations in Mainland China, Hong Kong, and Taiwan. Diabetes Res Clin Pract 2006;73:126-134.

27 Wee CC, Hamel MB, Huang A, Davis RB, Mittleman MA, McCarthy EP: Obesity and undiagnosed diabetes in the U.S. Diabetes Care 2008;31:18131815.

28 Lao XQ, Thomas GN, Jiang CQ, Zhang WS, Yin $\mathrm{P}$, Adab P, et al: Association of the metabolic syndrome with vascular disease in an older Chinese population: Guangzhou Biobank Cohort Study. J Endocrinol Invest 2006;29:989-996. 